Accepted to AJ

Preprint typeset using LATEX style AASTeX6 v. 1.0

\title{
ZODIACAL EXOPLANETS IN TIME (ZEIT) III: \\ A SHORT-PERIOD PLANET ORBITING A PRE-MAIN-SEQUENCE STAR IN THE UPPER SCORPIUS OB ASSOCIATION
}

\author{
Andrew W. Mann ${ }^{1,2}$, Elisabeth R. Newton ${ }^{3,4}$, Aaron C. Rizzuto ${ }^{1}$, Jonathan Irwin ${ }^{3}$, Gregory A. Feiden ${ }^{5}$ \\ Eric Gaidos ${ }^{6}$, Gregory N. Mace ${ }^{1}$, Adam L. Kraus ${ }^{1}$, David J. James ${ }^{7}$, Megan Ansdell ${ }^{8}$, David Charbonneau ${ }^{3}$, \\ Kevin R. Cover ${ }^{9}$, Michael J. Ireland ${ }^{10}$, Daniel T. Jaffe ${ }^{1}$, Marshall C. Johnson ${ }^{1}$, Benjamin Kidder ${ }^{1}$, Andrew \\ VANDERBURG $^{3,4}$ \\ ${ }^{1}$ Department of Astronomy, The University of Texas at Austin, Austin, TX 78712, USA \\ ${ }^{2}$ Hubble Fellow \\ ${ }^{3}$ Harvard-Smithsonian Center for Astrophysics, Cambridge, Massachusetts 02138, USA \\ ${ }^{4}$ NSF Graduate Research Fellow \\ ${ }^{5}$ Department of Physics and Astronomy, Uppsala University, Uppsala, Sweden \\ ${ }^{6}$ Department of Geology \& Geophysics, University of Hawaii at Manoa, Honolulu, HI 96822, USA \\ ${ }^{7}$ Cerro Tololo Inter-American Observatory, Colina el Pino, LaSerena, Chile \\ ${ }^{8}$ Institute for Astronomy, University of Hawaii at Manoa, Honolulu, HI 96822, USA \\ ${ }^{9}$ Western Washington University, Department of Physics \& Astronomy, Bellingham, WA 98225, USA \\ ${ }^{10}$ Research School for Astronomy \& Astrophysics, Australia National University, Canberra ACT 2611, Australia
}

\begin{abstract}
We confirm and characterize a close-in $\left(P_{\text {orb }}=5.425\right.$ days $)$, super-Neptune sized $\left(5.04_{-0.37}^{+0.34} \mathrm{R}_{\oplus}\right)$ planet transiting K2-33 (2MASS J16101473-1919095), a late-type (M3) pre-main sequence (11 Myr-old) star in the Upper Scorpius subgroup of the Scorpius-Centaurus OB association. The host star has the kinematics of a member of the Upper Scorpius OB association, and its spectrum contains lithium absorption, an unambiguous sign of youth $(<20 \mathrm{Myr})$ in late-type dwarfs. We combine photometry from K2 and the ground-based MEarth project to refine the planet's properties and constrain the host star's density. We determine K2-33's bolometric flux and effective temperature from moderate resolution spectra. By utilizing isochrones that include the effects of magnetic fields, we derive a precise radius $(6-7 \%)$ and mass $(16 \%)$ for the host star, and a stellar age consistent with the established value for Upper Scorpius. Follow-up high-resolution imaging and Doppler spectroscopy confirm that the transiting object is not a stellar companion or a background eclipsing binary blended with the target. The shape of the transit, the constancy of the transit depth and periodicity over 1.5 years, and the independence with wavelength rules out stellar variability, or a dust cloud or debris disk partially occulting the star as the source of the signal; we conclude it must instead be planetary in origin. The existence of K2-33b suggests close-in planets can form in situ or migrate within $~ 10 \mathrm{Myr}$, e.g., via interactions with a disk, and that long-timescale dynamical migration such as by Lidov-Kozai or planet-planet scattering is not responsible for all short-period planets.
\end{abstract}

Keywords: stars: fundamental parameters - stars: individual (K2-33) — stars: late-type — stars: low-mass - stars: planetary systems — stars: young

\section{INTRODUCTION}

Many known exoplanets orbit within $0.1 \mathrm{AU}$ of their host star, where they are more readily detected by the transit and Doppler methods (e.g., Howard et al. 2010; Fressin et al. 2013). Whether these planets formed near their present position (in situ), i.e., from circumstellar material interior to $0.5 \mathrm{AU}$ (e.g., Chiang \& Laughlin 2013; Ogihara et al. 2015), or accreted at distances > $1 \mathrm{AU}$, and later migrated inwards (e.g., Schlaufman et al.
2009; Raymond \& Cossou 2014) is actively debated. If the planets migrated, the physical mechanism(s) behind their migration is yet another further point of debate.

Mechanisms of planet migration can be loosely divided into three categories: interactions with the protoplanetary disk (e.g., Ida \& Lin 2008; Lubow \& Ida 2010), interactions between a stellar companion and the planet (the Lidov-Kozai mechanism, e.g., Wu \& Murray 2003), and interactions among multiple planets (e.g., Ford \& Ra- 
sio 2006; Chatterjee et al. 2008). Disk migration must occur before the protoplanetary disk dissipates/photoevaporates ( $\lesssim 10 \mathrm{Myr})$ (e.g., Ward 1997). Migration involving angular momentum exchange with a third body typically operates on timescales much longer than disk migration $(\simeq 100 \mathrm{Myr}$ to more than $1 \mathrm{Gyr}$ ), depending on the orbital and physical properties of the planet and perturber (Fabrycky \& Tremaine 2007; Nagasawa et al. 2008).

The difference in timescales presents a possible method to distinguish between these migration mechanisms. Close-in super-Earths or Jupiter-size planets around stars younger than $100 \mathrm{Myr}$ could not have migrated by a slow process like planet-planet or planet-star interaction, and instead likely formed in situ or migrated quickly through interaction with the disk. A comparison of the occurrence rate of close-in planets over a range of ages (10-1000 Myr) would constrain the fraction of planets migrating on a given timescale.

High-precision photometry offers the best opportunity to detect the close-in planets needed to test migration theories (e.g., Janes 1996). Such planets are too close to their host star to be detected by direct imaging (e.g., Le Bouquin \& Absil 2012; Bowler et al. 2015b). Starspotinduced jitter complicates the detection of the planetary reflex motion (Mahmud et al. 2011; Paulson et al. 2004), such that radial velocity (RV) surveys of young stars primarily uncover hot Jupiters around stars older than 100 Myr (e.g., Quinn et al. 2012a, 2014). Spot modulation can generate complicated variations in the light curve that makes detecting transiting planets more difficult. However, spots and transits create characteristically different patterns in a light curve which can be separated with precise photometry. Indeed the only close-in planet (candidates) around $<20 \mathrm{Myr}$ old stars are from transit surveys (van Eyken et al. 2012; Mamajek et al. 2012; Kenworthy et al. 2015, e.g.,).

The repurposed Kepler mission (K2, Howell et al. 2014) has the photometric precision (tens of ppm) and observational baseline (70-80 days) required to detect small planets and rule out false-positive signals related to stellar youth (e.g., debris disks, and spots). We are carrying out a search for planets transiting stars in 10800 Myr young open clusters and OB associations using K2. Our survey, Zodiacal Exoplanets in Time, includes Upper Scorpius ( $\simeq 11 \mathrm{Myr}$, Pecaut et al. 2012; Rizzuto et al. 2016), Taurus (Kenyon et al. 2008, 0-5 Myr, ), Pleiades ( $125 \mathrm{Myr}$, Dahm 2015), and Praesepe and Hyades (650-800 Myr, Brandt \& Huang 2015). Our goal is to better understand if planets evolve from infancy (1-10 Myr) to maturity ( $\gtrsim 1 \mathrm{Gyr}$ ), including how planets migrate, how, and on what timescales.

Here we confirm and characterize a $5.04_{-0.37}^{+0.34} \mathrm{R}_{\oplus}$ planet (K2-33b) orbiting the pre-main-sequence
(PMS) star K2-33 (2MASS J16101473-1919095, EPIC 205117205), a member of the Upper Scorpius subgroup of the Scorpius-Centaurus (Sco-Cen) OB association. K2-33b was previously identified as a planet candidate by Vanderburg et al. (2016), but assigned inaccurate stellar and planetary parameters owing to the assumption of a main sequence age and an unreddened spectral energy distribution for the host star. In Section 2 we describe our follow-up observations, including moderate- to high-resolution spectroscopy, adaptive optics imaging and non-redundant aperture masking, and transit photometry. Our analysis of the light curve data is described in Section 3. In Section 4 we derive parameters for the host star. We use the available data to confirm a planetary origin of the transit signal, as we describe in Section 5. We conclude in Section 6 with a brief summary and discussion of the importance of this planet.

\section{OBSERVATIONS AND DATA REDUCTION}

\subsection{K2 observations and light curve extraction}

From 2014 August 23 to 2014 November 13 (Campaign 2) K2 observed the core of Upper Scorpius, including K2-33. Owing to the loss of two reaction wheels the Kepler satellite drifts. To correct the pointing, Kepler's thrusters fire every $\sim 6$ hours. However, during the drift and subsequent thruster fire a stellar image usually moves over the detector. Combined with variations in the pixel sensitivity this generates changes in total measured flux from a given star as a function of centroid position.

Vanderburg \& Johnson (2014) present a method for mitigating or removing this noise, however it is not optimized for highly variable stars, e.g., the young stars of Upper Scorpius. The transit can still be identified in the Vanderburg \& Johnson (2014) light curve, but systematic trends are present, including a discontinuity in the middle of the observations when Kepler changed the direction of its roll, and large changes in the point-topoint scatter over the observing window due to poor treatment of the thruster-fire systematics. Following Becker et al. (2015) and Mann et al. (2016) we extracted a new light curve by simultaneously fitting for low frequency variations from stellar activity, Kepler flat field, and the transits of K2-33b using a least-square minimization. Both stellar variability and the effect of errors in detector response were both modeled as splines as a function of time and centroid position with breakpoints every 0.2 days and $0.4^{\prime \prime}$, respectively. Unlike Vanderburg et al. (2016), we did not apply separate systematics corrections to the first and second half of the $K 2$ campaign, which removed the major discontinuity. The resulting light curve is relatively clear of visible systematic errors 
(see Figure 1).

\subsection{Optical Spectrum from SNIFS}

We obtained an optical spectrum of K2-33 on February 23, 2016 (UT) with the SuperNova Integral Field Spectrograph (SNIFS, Aldering et al. 2002; Lantz et al. 2004) on the University of Hawai'i $2.2 \mathrm{~m}$ telescope on Maunakea. SNIFS covers 3200-9700 ̊ simultaneously with a resolution of $R \simeq 700$ and $R \simeq 1000$ in the blue (3200-5200 $\AA$ ) and red (5100-9700 $\AA$ ) channels, respectively. A single 710 s exposure yielded $\mathrm{S} / \mathrm{N}=80$ per resolving element in the red channel. We also observed 5 spectrophotometric standards throughout the night for flux calibration. ThAr arcs were taken before or after each observation to improve the wavelength solution. Bias, flat and dark correction, as well as cosmic ray rejection, construction of the data cubes, and extraction of the one-dimensional spectrum are described in detail in Aldering et al. (2002). The flux calibration is derived from the combination of the spectrophotometric standards and a model of the atmospheric absorption above Maunakea as described in Mann et al. (2015).

\subsection{NIR Spectrum from ARCoIRIS}

During the night of January 25, 2016 (UT), we acquired $z^{\prime} Y J H K$ spectra $(\simeq 0.8-2.45 \mu \mathrm{m})$ of K2-33 using the ARCoIRIS spectrograph (Schlawin et al. 2014), newly installed at the Cassegrain focus of the Blanco $4 \mathrm{~m}$ telescope at the Cerro Tololo InterAmerican Observatory. ARCoIRIS is a fixed-format, cross-dispersed, long-slit spectrograph projected onto an HAWAII-2RG array having $18-\mu \mathrm{m}$ pixels. We used its $110.5 \mathrm{l} \mathrm{mm}^{-1}$ reflection grating and a $1.1 " \times 28$ " slit to obtain an approximate spectral resolution of $R \simeq 3500$ across all six spectral orders.

We placed the object at two widely separated positions along the slit, A and B, and took two series of ABBA nods, with per-nod position exposure times of $100 \mathrm{~s}$. Immediately afterwards, we took a similar series of ABBA nod observations for the A0V standard HD 146606. An accompanying $\mathrm{Cu}-\mathrm{He}-\mathrm{Ar}$ comparison lamp spectrum was also obtained for wavelength calibration. Data reduction of the target and calibrator was performed using the SpeXtool suite of IDL packages (version 4.1, Cushing et al. 2004) adapted for the data format and characteristics of the ARCoIRIS instrument (priv comm. Katelyn Allers ${ }^{1}$ ). Each difference (A-B) image was flat-fielded, wavelength calibrated, and extracted to produce a one-dimensional spectra. The telluric calibrator star (HD 146606) was used to telluric correct and flux calibrate the target spectrum (employing the pack- age xtellcorr; Vacca et al. 2004). The final reduced and stacked spectrum has a peak $\mathrm{S} / \mathrm{N}>200$ per resolving element in the $H$ and $K$ bands.

\subsection{High Resolution NIR Spectrum}

We observed K2-33 on the nights of January 30, February 26, March 28 and 29, 2016 (UT) with the Immersion Grating Infrared Spectrometer (IGRINS, Park et al. 2014) on the $2.7 \mathrm{~m}$ Harlan J. Smith telescope at McDonald Observatory. IGRINS provides simultaneous $H$ - and $K$-band (1.48-2.48 $\mu \mathrm{m})$ coverage with a resolving power of $R \simeq 45,000$. Similar to the ARCoIRIS observations, the target was placed at two positions along the slit (A and B) and observed in an ABBA pattern. Each integration was $600 \mathrm{~s}$, which, when stacked, yielded a $\mathrm{S} / \mathrm{N}=50$ 80 per resolving element near the center of each spectral order (at each of the four epochs).

The IGRINS spectra were reduced using version 2.1 of the publicly available IGRINS pipeline package ${ }^{2}$ (Lee 2015), which provides optimally extracted onedimensional spectra of both the A0V standard and target. We used the A0V spectra to correct for telluric lines following the method outlined in Vacca et al. (2003). Spectra without telluric correction were kept and used to improve the wavelength solution and provide a zeropoint for the RVs.

Radial velocities were determined from the IGRINS data as explained in Mann et al. (2016) and Mace et al. (in prep). In brief, we used the telluric lines like an iodine cell to lock the wavelength solution over epochs months apart, then cross-correlated the spectrum against 230 RV templates with spectral type M0-M6. The final assigned RV and error is the robust mean and standard error of the cross-correlation across all 230 templates. The absolute RV was taken to be the weighted mean of the four individual measurements, with an error limited by the zero-point error of $153 \mathrm{~m} \mathrm{~s}^{-1}$. Relative RV errors are generally $40 \mathrm{~ms}^{-1}$, except for the first epoch, which had unusually high telluric contamination and lower S/N.

\subsection{Adaptive Optics Imaging and Aperture Masking}

On March 18 (UT), 2016 we obtained natural guide star adaptive optics (AO) imaging (Wizinowich et al. 2000) and non-redundant aperture masking (NRM) of K2-33 with the facility imager, NIRC2, on Keck II atop Maunakea. All observations were taken in vertical angle mode, using the smallest pixel scale $(9.952 \pm 0.002$ mas/pix). Imaging was taken with the $K^{\prime}$ filter and masking with the 9-hole mask. After AO loops closed we took eight images, each with 20 coadds and an integration time of $0.5 \mathrm{~s}$ per coadd. For NRM we took 10

\footnotetext{
${ }^{2}$ https://github.com/igrins/plp 


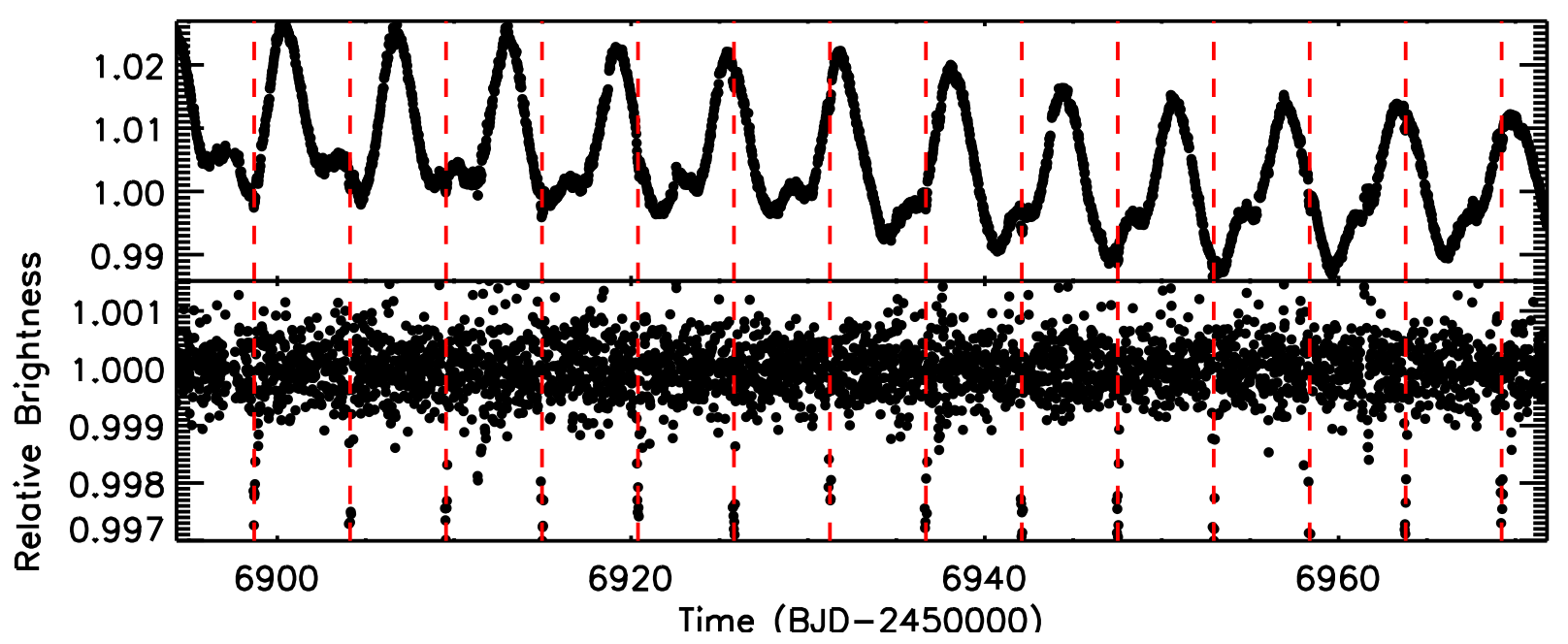

Figure 1. Light curve of K2-33 taken by the Kepler spacecraft. The top panel displays the light curve constructed from Kepler pixel data after removing effects of Kepler roll. The bottom panel shows the light curve after removing stellar variability. Red dashed lines indicate transits. Both curves are normalized to one.

interferograms, each with an integration of time of $20 \mathrm{~s}$ and 1 coadd.

Each frame was linearized and corrected for geometric distortion using the NIRC2 solution from Yelda et al. (2010). Images were dark-subtracted and flat-fielded. We interpolated over "dead" and "hot" pixels. Dead pixels were identified from superflats taken in 2006-2013 as any pixel with a response of $<30 \%$ in at least half of all superflats. Similarly, hot pixels were identified from a comparable set of superdarks as any pixel with $\geq 10$ counts in at least half of the superdarks. Pixels with flux levels $>10 \sigma$ above the median of the 8 adjacent pixels were flagged as cosmic rays or transient hot pixels and replaced with the median.

To detect faint and wide ( $~ 500$ mas) companions in the AO images we subtracted an azimuthal median PSF model built from the smoothed PSF of K2-33. This added no additional noise at wide separations, but left the speckles in place, making it non-ideal for detecting close-in companions. To probe smaller inner working angles we instead constructed and subtracted the bestfitting PSF of another (single-star) target taken in the same night. We stacked all subtracted frames of K233 , and identified companions by measuring the flux in 40 mas (radius) apertures centered on every image pixel. We measured our detection limits from the standard deviation of the flux among all apertures in a 5-pixel annulus around the primary. We found no apertures that contained sufficient flux within the NIRC2 field of view to be considered an astrophysical source.

The aperture masking observations use the complex triple product, or closure-phase, to remove non-common path errors introduced by atmospheric conditions and

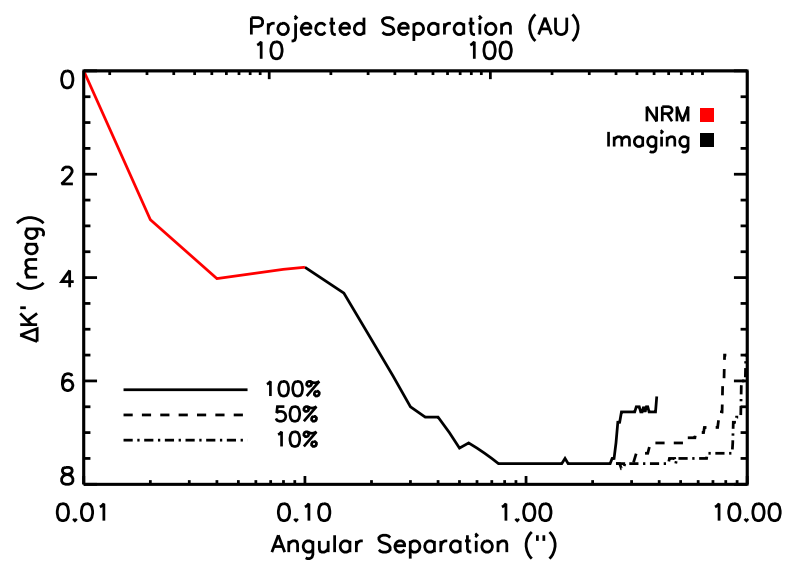

Figure 2. Detection limits $(5 \sigma)$ for K2-33 from our AO imaging and NRM interferometry as a function of separation. The top axis shows the separation in AU assuming a distance of $145 \mathrm{pc}$. The region probed by non-redundant aperture masking is marked in red, while the region probed by imaging is in black. Owing to the finite chip size data is incomplete as a function of azimuthal angle at large separations. There we show the contrast curves with $100 \%$ (solid line), $50 \%$ (dashed), and 10\% (dotted-dashed) azimuthal completeness.

variable optical aberrations. To remove systematics, the observation of K2-33 was paired with a calibration observation of USco J160933.8-190456, another member of Upper Scorpius (Preibisch et al. 2001). Binary system profiles can then be fit to the closure phases to produce separations and position angles and calculate contrast limits. The appendix of Kraus et al. (2008) contains a full explanation of the data reduction and binary profilefitting for aperture masking data. No sources were detected in the masking data.

The combination of the aperture masking and imaging 
observations excludes contributions of additional stars at separations 0.02-3" to the lightcurve. Figure 2 displays the imaging and masking contrast limits as a function of separation. Owing to the edges of the detector the azimuthal coverage is not complete outside of $\simeq 3^{\prime \prime}$.

\subsection{Transit photometry from MEarth}

We observed two additional transits of K2-33b using the MEarth-North and MEarth-South arrays (Nutzman \& Charbonneau 2008; Berta et al. 2012; Irwin et al. 2015) on February 16, 2016 and on March 14, 2016 (UT). MEarth-North comprises eight 40-cm telescopes at Fred Lawrence Whipple Observatory on Mount Hopkins, Arizona. MEarth-South uses a nearly identical set of telescopes located at Cerro Tololo Inter-American Observatory $($ CTIO) in Chile. All telescopes use a $2048 \times 2048$ pixel CCD with pixels scales of $0.78^{\prime \prime} /$ pixel in the north and $0.84^{\prime \prime} /$ pixel in the south. The Schott RG715 filter was used for all observations (see Dittmann et al. 2016, for the filter profile and CCD transmission).

All telescopes integrated for $60 \mathrm{~s}$ for a cadence of $\simeq 90 \mathrm{~s}$ per telescope. The first transit observation was only visible from MEarth-South, during which four telescopes simultaneously observed the second half of the transit, including $\gtrsim 1$ hour after egress to fit for stellar variability. The second observation included the full transit, the first half of which was observed by four telescopes at MEarth-South and the second half by six telescopes in MEarth-North (including $\simeq 30 \mathrm{~m}$ of simultaneous observations). Each array monitored K2-33 for at least an hour of before or after the transit. In total more than 1200 photometric measurements were taken by MEarth during these transits.

MEarth also took low-cadence photometry of K233 from January 26, 2016 to March 26, 2016 to constrain the long-term photometric variability. This longterm monitoring was done with one of the MEarthSouth telescopes, which took two $60 \mathrm{~s}$ exposures every 20-30 minutes whenever the target was visible and the weather was amenable.

MEarth data were reduced following the basic methodology from Irwin et al. (2007) with additional steps detailed in the documentation of the fourth MEarth data release $^{3}$. This included corrections for second order extinction (color differences between target and comparison stars), meridian flips (when the target crosses the meridian the telescope rotates by 180 degrees relative to the sky, and reference stars fall on different parts of the detector), and stellar variability (fit from the transit and long-term monitoring). We also removed data points with anomalously high errors $(>0.8 \%$, mostly

3 https://www.cfa.harvard.edu/MEarth/DR4/processing/index.html taken during twilight). Lastly, we scaled the flux from the two telescope arrays to force agreement between the overlapping data.

\section{LIGHT CURVE ANALYSIS}

\subsection{Transit Identification}

The transit around K2-33 was initially identified by Vanderburg et al. (2016) as an Earth-sized planet orbiting an $\mathrm{M}$ dwarf $\left(T_{\mathrm{eff}}=2890 \mathrm{~K}, R_{*}=0.16 R_{\odot}\right)$ every 5.425 days. Vanderburg et al. (2016) assumed the host star was unreddened, causing the inferred $T_{\text {eff }}$ to be erroneously low. Further, the assumption that the star was on the main sequence led to an even more erroneously low inferred stellar radius. Thus the estimated radius of the planet was under-estimated as well.

The transit signal of K2-33 was independently identified by the ZEIT project (Mann et al. 2016) and the Mass-Radius Relation of Young Stars (Kraus et al. 2015), both while searching for transiting/eclipsing systems in Upper Scorpius. The ZEIT search method is based on the box-least squares algorithm (Kovács et al. 2002), but optimized for high amplitude rapid rotators. K2-33 was the first Upper Scorpius planet candidate identified by our search, as it exhibits a comparatively high $\mathrm{S} / \mathrm{N}(\simeq 25)$ trapezoidal signal with a long-duration $(\simeq 4 \mathrm{~h})$ as expected for a planet around a still contracting, PMS star.

\subsection{Transit fitting}

We simultaneously fit the $K 2$ and MEarth transit data with a Monte Carlo Markov Chain (MCMC) as described in Mann et al. (2016), which we briefly summarize here. We used the emcee Python module (ForemanMackey et al. 2013) to fit the model lightcurves produced by the batman package (Kreidberg 2015) using the Mandel \& Agol (2002) algorithm. Following Kipping (2010) we over-sampled and binned the model to match the 30 minute $K 2$ cadence. We used an unbinned model to fit the MEarth data due to the much lower integration time $(60 \mathrm{~s})$. We sampled the planet-to-star radius ratio $\left(R_{P} / R_{*}\right)$, impact parameter $(b)$, orbital pe$\operatorname{riod}(P)$, epoch of the first transit mid-point $\left(T_{0}\right)$, bulk stellar density $\left(\rho_{*}\right)$, and two limb darkening parameters ( $q 1$ and $q 2$ ) for each of the two instruments (MEarth and $K 2)$. At this young age it is likely that any eccentricity was dampened by the primordial disk (e.g., Tanaka \& Ward 2004; Cresswell et al. 2007), so we fix the eccentricity at zero. However, the eccentricity distribution of young planets has not been observationally constrainted; we discuss lifting this assumption in Section 6 .

We assumed a quadratic limb darkening law and used the triangular sampling method of Kipping (2013) in order to uniformly sample the physically allowed region of parameter space. We applied a prior on limb darkening 
derived from the Husser et al. (2013) atmospheric models, calculated using the LDTK toolkit (Parviainen \& Aigrain 2015), which enabled us to account for errors in stellar parameters and finite grid spacing. Stellar parameters input into LDTK are derived in Section 4. Errors on the limb darkening coefficients are broadened by a factor of two to account for model uncertainties (estimated by comparing limb darkening parameters from different model grids). The filter profiles and CCD transmission functions were taken from Dittmann et al. (2016) for the MEarth bandpass and from the Kepler science center ${ }^{4}$ for the Kepler bandpass. This yielded quadratic limb darkening coefficients of $\mu_{1}=0.4 \pm 0.1$ and $\mu_{2}=0.4 \pm 0.1$ for Kepler and $\mu_{1}=0.26 \pm 0.09$ and $\mu_{2}=0.4 \pm 0.1$ for MEarth.

Our MCMC was allowed to explore $|b|<1+R_{P} / R_{*}$, $P$ from 0 to 70 days, $\rho_{*}$ from 0 to $\infty, R_{P} / R_{*}$ from 0 to 1 , and $T_{0}$ from \pm 3 days from the initial value, all with uniform priors. All parameters were initialized to the values from our BLS search (Section 3.1), which are based on a Levenberg-Marquardt fit to the light curve (Markwardt 2009). MCMC chains were run using 150 walkers, each with 100,000 steps after a burn-in phase of 5,000 steps.

We report the transit fit parameters in Table 1. For each parameter we report the median value with the errors as the 84.1 and 15.9 percentile values (corresponding to $1 \sigma$ for Gaussian distributions). The model light curve with the best-fit parameters is shown in Figure 3 alongside the K2 and MEarth data. Some correlated errors are present in the MEarth light curve, primarily during ingress, which we attribute to imperfect correction of stellar variability and/or the planet crossing a spot. We also show posteriors and correlations for a subset of parameters in Figure 4.

The transit posterior favors a low $(<0.4)$ impact parameter, although there is a tail in the distribution corresponding to higher impact parameter, lower $\rho_{*}(<0.3)$, and larger planet radius. This region of parameter space is not ruled out by our independent stellar parameters (see Section 4), so we did apply an additional constraint in the MCMC or remove these solutions from our transitfit posterior.

\section{STELLAR PARAMETERS}

Membership in Upper Scorpius: The spatial position and kinematics of K2-33 are consistent with co-motion of the star with the ensemble Upper Scorpius space velocity. We calculate a photometric distance to K2-33 of $140 \pm 16 \mathrm{pc}$ using literature optical and NIR photometry, and the $10 \mathrm{Myr}$ isochrone from Chen et al. (2014).

4 http://keplergo.arc.nasa.gov/CalibrationResponse.shtml

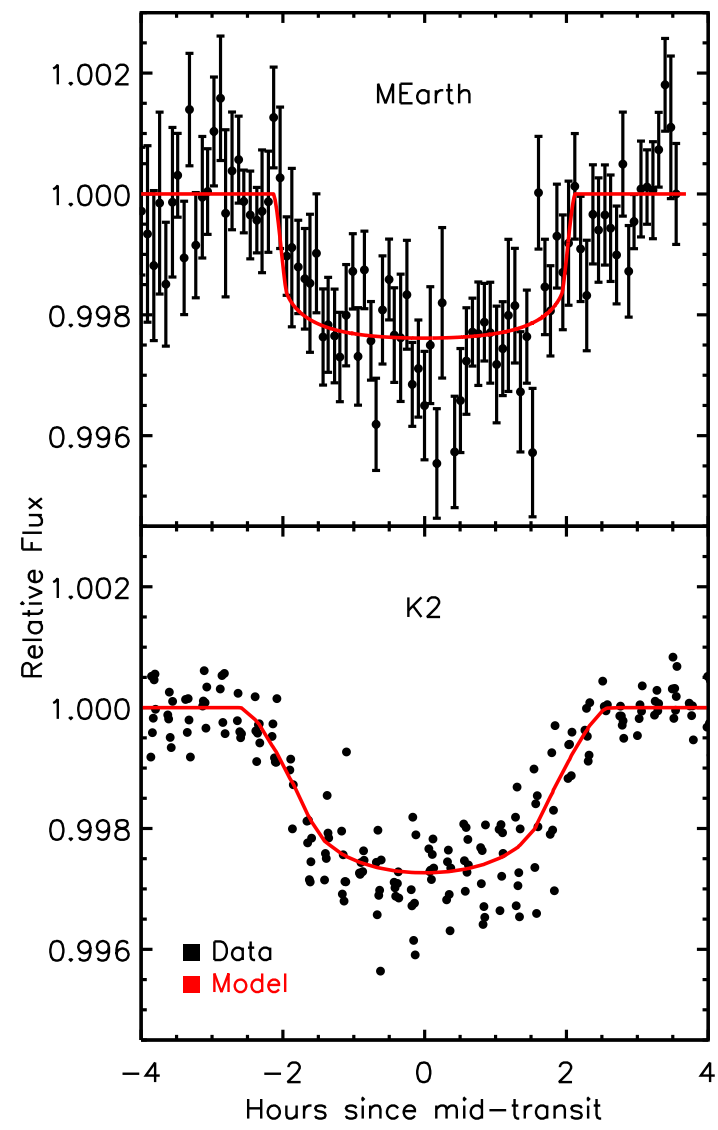

Figure 3. Phase-folded light curve of K2-33's transit (black) from MEarth (top, binned) and K2 (bottom). The best-fit transit models are show in red. Owing to the large number of data points from MEarth we bin every 5 min of data and show the median and $1 \sigma$ scatter of points in each bin (unbinned data is used for MCMC fit). The $K 2$ fit hasa longer ingress/egress because of the $30 \mathrm{~min}$ integration time, which is accounted for in the model. Some systematics are present in the ingress of the MEarth transit, which we attribute to imperfect correction of stellar variability.

This is consistent with the Hipparcos distances to highmass members of Upper Scorpius ( $145 \pm 15$ pc, de Zeeuw et al. 1999; Rizzuto et al. 2011). Using this photometric distance, proper motions from UCAC4 $(-9.8 \pm 1.7$, $24.2 \pm 1.8$ mas yr$^{-1}$ Zacharias et al. 2012), and the mean RV from our IGRINS observations, we calculate the Galactic space velocity of $\mathrm{K} 2-33(U, V, W)=(5.4 \pm 0.5$, $\left.-15.8 \pm 2.2,-8.2 \pm 1.2 \mathrm{~km} \mathrm{~s}^{-1}\right)$. This is consistent with the kinematic models of Chen et al. (2014) and velocity dispersion of $\sim 2-3 \mathrm{~km} / \mathrm{s}$ (Kraus \& Hillenbrand 2008) for Upper Scorpius. Using the Bayesian method from Rizzuto et al. (2011) and Rizzuto et al. (2015) we calculate a probability of membership in Upper Scorpius of $96 \%$ for K2-33.

K2-33 also shows multiple indicators of youth. We measured the Na-8189 index, a well-calibrated gravity index (Slesnick et al. 2006) to be $0.946 \pm 0.005$, consis- 
Table 1. Transit Fit Parameters

\begin{tabular}{ll}
\hline \hline \multicolumn{1}{c}{ Parameter } & \multicolumn{1}{c}{ Value } \\
\hline Period (days) & $5.424865_{-0.000031}^{+0.000035}$ \\
$R_{P} / R_{*}$ & $0.0432_{-0.0007}^{+0.0009}$ \\
$\mathrm{~T}_{0}{ }^{\mathrm{a}}$ (BJD-2400000) & $56898.69288_{-0.00120}^{+0.00118}$ \\
Density $\left(\rho_{\odot}\right)$ & $0.51_{-0.07}^{+0.04}$ \\
Impact Parameter & $0.16_{-0.11}^{+0.19}$ \\
Duration (hours) & $4.08_{-0.07}^{+0.07}$ \\
$a / R_{*}$ & $10.40_{-0.50}^{+0.27}$ \\
Inclination (degrees) & $89.1_{-1.1}^{+0.6}$ \\
Eccentricity & $0($ fixed) \\
$\omega($ degrees) & 0 (fixed) \\
\hline$R_{P}{ }^{\mathrm{b}}$ (R $\left.\mathrm{R}_{\oplus}\right)$ & $5.04_{-0.37}^{+0.34}$ \\
\hline$a_{\mathrm{BJD} \text { is in Barycentric Dynamical Time }}$ \\
(TBD) format. \\
$b_{\text {Planet radius is derived using our stellar ra- }}$ \\
dius (Section 4).
\end{tabular}

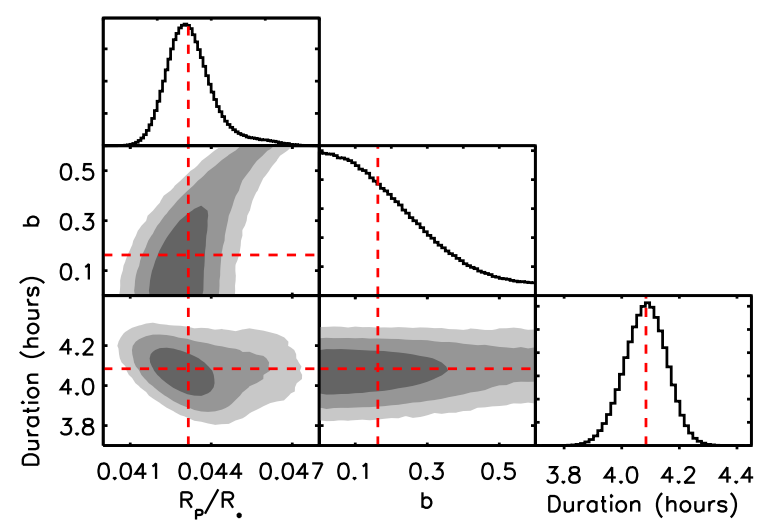

Figure 4. Posteriors (histograms) and parameter correlations (contour plots) from our MCMC fit to the MEarth and K2 light curves. Median values for each parameter are marked with red dashed lines. Grey shading covers $67 \%, 95 \%$ and $99 \%$, from dark to light, of the MCMC posterior.

tent with other late-type stars in Upper Scorpius and suggesting an age of 5-30 Myr. K2-33 shows a $24 \mu \mathrm{m}$ excess (Luhman \& Mamajek 2012) in Spitzer:MIPS observations, suggesting the presence of a disk, and hence an age for K2-33 of <40 Myr. Further, K2-33 was already identified as a member of the Upper Scorpius subgroup by the presence of a strong $\mathrm{Li} 6708 \AA$ line $(0.45 \pm 0.15 \AA$, Preibisch et al. 2001), an unambiguous indicator of youth for late-type stars.

Spectral type and reddening: following Kraus et al. (2015) and Ansdell et al. (2016) we simultaneously solved for spectral type and reddening $\left(A_{V}\right)$ to account for correlations between these parameters. We compared our optical spectrum of K2-33 to a grid of 270 unreddened optical spectra of young stars from Herczeg \& Hillenbrand (2014). For each template we computed the $A_{V}$ value that gives the best agreement between the spectrum of K2-33 and that of the template using the reddening law from Cardelli et al. (1989) and masking out the $\mathrm{H}-\alpha$ line and the strong $\mathrm{O}_{2}$ tellurics. The resulting distribution of reduced $\chi^{2}\left(\chi_{\nu}^{2}\right)$ values yielded a spectral type of M3.3 \pm 0.2 with an $A_{V}$ of $0.75_{-0.18}^{+0.21}$ (Figure 5). This spectral type error does not account for systematic errors in the spectral typing scheme (which can vary by $0.5-1$ spectral subtype between methods), so we instead we adopted a more conservative M3.3 \pm 0.5 . This does not affect the $A_{V}$ determination, but $A_{V}$ could be affected if there are systematic errors in the spectrophotometric calibration of our optical spectrum or the Herczeg \& Hillenbrand (2014) templates.

To test our sensitivity to our choice of templates, we repeat the above process with $\mathrm{M}$ dwarf spectral templates from Gaidos et al. (2014). These were taken with the same instrument as our spectrum of K2-33, but the spectra are predominantly from old ( $>1 \mathrm{Gyr}$ ) stars. The Gaidos et al. (2014) templates give a slightly earlier spectral type and higher reddening (M3.1, 0.83), but both are consistent with the values derived using templates from young stars. Comparison to the Gaidos et al. (2014) templates also give significantly higher $\chi_{\nu}^{2}$ values than to those from Herczeg \& Hillenbrand (2014), likely because of gravity-dependent differences in the spectrum. 

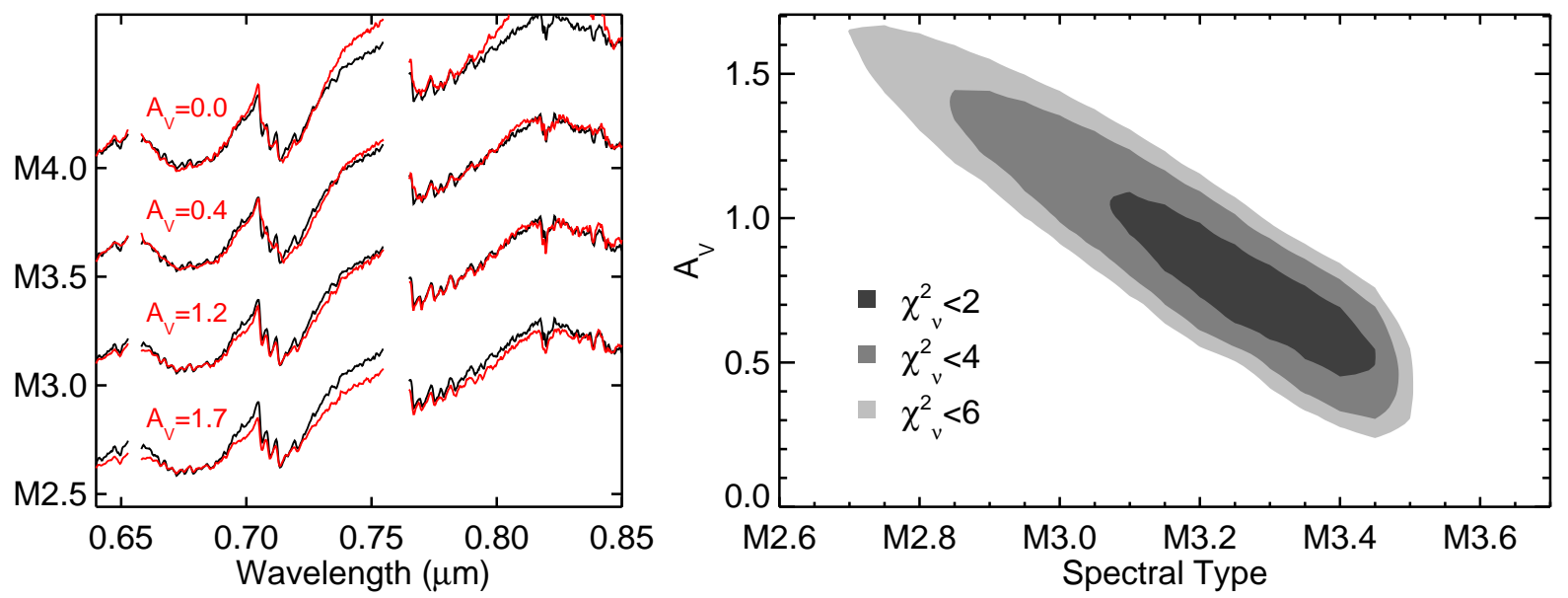

Figure 5. Left: Optical spectrum of K2-33 (black) compared to a M2.5-M4 young templates from Herczeg \& Hillenbrand (2014), which are shown in red. For each template we found the best-fit $A_{V}$ value (lowest $\left.\chi^{2}\right)$. Right: the reduced $\chi^{2}\left(\chi_{\nu}^{2}\right)$ surface as a function of the template spectral type and reddening. This suggests a best-fit spectral type of M3.3 \pm 0.2 with an $A_{V}$ of $0.75_{-0.18}^{+0.21}$.

Effective Temperature: We compared the unreddened spectrum to a grid of BT-SETTL CIFIST models ${ }^{5}$ (Allard et al. 2011, 2012), masking our regions where models poorly reproduce observed spectra and accounting for small errors in the flux and wavelength solution as detailed in Mann et al. (2013) and Gaidos et al. (2014). This method has been shown to reproduce $T_{\text {eff }}$ values for main-sequence $\mathrm{M}$ dwarfs derived from interferometry (Boyajian et al. 2012), but is poorly tested on PMS stars. However, we accurately reproduced the geometric $T_{\text {eff }}$ derived for the low-mass eclipsing binary USco CTIO5 (Kraus et al. 2015) suggesting our method yields reasonable $T_{\text {eff }}$ values even at young ages. To account for errors in reddening we repeated this process over the range of reddening values derived above. The effect of reddening is small, as the model comparison is driven primarily by the depth of the molecular bands instead of the overall spectral shape. We found a best-fit $T_{\text {eff }}$ of $3540 \pm 70 \mathrm{~K}$

Bolometric Flux: we compiled optical BVgri photometry from the ninth data release of the AAVSO AllSky Photometric Survey (APASS, Henden et al. 2012), NIR $J H K_{S}$ photometry from The Two Micron All Sky Survey (2MASS, Skrutskie et al. 2006), griz photometry from the Sloan Digital Sky Survey (SDSS, Ahn et al. 2012), and $W 1 W 2 W 3$ infrared photometry from the Wide-field Infrared Survey Explorer (WISE, Wright et al. 2010). We then scaled the (reddened) NIR and optical spectrum to the archival photometry following the procedure from Mann et al. (2015). The flux-calibrated spectrum is plotted in Figure 6. We then unredden the

\footnotetext{
5 https://phoenix.ens-lyon.fr/Grids/BT-Settl/CIFIST2011
}

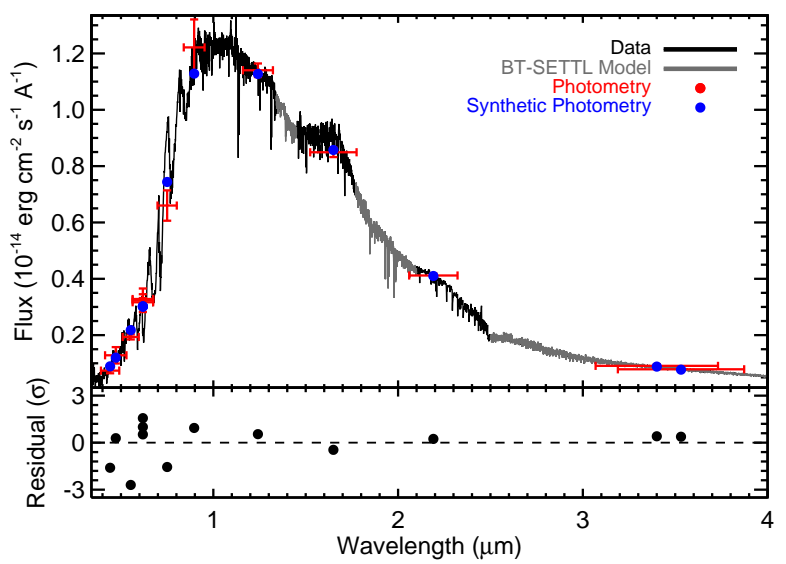

Figure 6. Absolute flux calibrated spectrum of K2-33. Archival photometry is shown in red, with the horizontal error bars representing the effective width of the filter. Synthetic photometry derived from convolving the spectrum with the appropriate filter profile and zero-point (Cohen et al. 2003; Jarrett et al. 2011; Mann \& von Braun 2015) are shown in blue. We replace regions of high telluric absorption and those outside the range of our empirical spectra with an atmospheric model, which we show in grey. The spectrum and photometry shown here have not been corrected for reddening. The bottom pane shows the residual (photometrysynthetic) in units of standard deviations.

calibrated spectrum. To calculate $F_{\mathrm{bol}}$ we integrated the spectrum over all wavelengths. As with $T_{\text {eff }}$, this process was repeated over the range of $A_{V}$ values, which effectively tripled our error on $F_{\text {bol }}$. We found a best-fit for $F_{\text {bol }}$ of $2.25( \pm 0.26) \times 10^{-10} \mathrm{erg} \mathrm{cm}^{-2} \mathrm{~s}^{-1}$.

Stellar radius, mass and age: we combined the distance to Upper Scorpius ( $145 \pm 15 \mathrm{pc}$, de Zeeuw et al. 1999) with our measured $F_{\text {bol }}$ and $T_{\text {eff }}$ to calculate the stellar radius from the Stefan-Bolzman relation. This gives a radius of $1.02 \pm 0.13 R_{\odot}$. We combined this with 
our transit-fit density from Section 3.2 to get a mass estimate of $0.55_{-0.14}^{+0.13} M_{\odot}$. However, we can significantly improve on these parameters, and also estimate the age of the system using a grid of PMS stellar evolution models.

We comparing our observables to two grids of PMS models computed with an updated version of the Dartmouth stellar evolution code (Dotter et al. 2008; Feiden \& Chaboyer 2012). A number of improvements to the code that allow more accurate computation of PMS stars are summarized in Feiden (2016). Germane to K2-33, one of the grids includes effects of magnetic inhibition of convection (Feiden \& Chaboyer 2012, 2013), which relieves the observed age discrepancy between early- and late-type stars in Upper Scorpius (Pecaut et al. 2012; Rizzuto et al. 2015), yielding a consistent 9-10 Myr median age for spectral types A through M (Feiden 2016).

To infer the mass, radius, and age of K2-33, an MCMC method implemented with emcee (ForemanMackey et al. 2013) was used to sample the parameter space covered by our two model grids. The nonmagnetic, standard model grid covered a mass range of 0.1-0.9 $M_{\odot}$ with a resolution of $0.02 M_{\odot}$ and a metallicity range of -0.5 to +0.5 dex at a resolution of $0.1 \mathrm{dex}$. The magnetic model grid covered a larger mass range of 0.1-1.7 $M_{\odot}$ with a resolution of $0.02 M_{\odot}$, but the metallicity was restricted to $[\mathrm{M} / \mathrm{H}]=0.0$ dex. We explored mass, metallicity (for non-magnetic models), age, and distance to find an optimal fit to the observables: $T_{\text {eff }}$ and $F_{\text {bol }}$, using the likelihood function given in Mann et al. (2015).

We applied a Gassian prior on distance $(145 \pm 15$ pc; de Zeeuw et al. 1999), a prior on $\rho_{*}$ drawn from our transit fit posterior (Section 3.2), and uniform priors on mass and age. For the non-magnetic models we applied a Gaussian prior on metallicity ( $0.0 \pm 0.1 \mathrm{dex}$; Bubar et al. 2011; Mamajek et al. 2013). To test the robustness of the results we also ran chains with a uniform prior on $\rho_{*}$ or distance (restricted to $10-1000 \mathrm{pc}$ ) for each of the model grids.

The MCMC simulation was set up with 300 walkers at random initial starting positions and was allowed to run over 1000 iterations following a burn-in phase of 250 iterations. Convergence was diagnosed through a combination of visually monitoring trace plots for all 300 chains, monitoring the median acceptance fraction among all chains (between $25 \%$ and $50 \%$ ), and by monitoring the auto-correlation time for individual chains. A summary of all MCMC results is given in Table 2. Quoted values represent the median of the posterior and quoted uncertainties are the $68 \%$ Bayesian credible intervals.

All chains produced consistent stellar radii, luminosities, and masses. This is in large part a consequence of the observables and constraints; $T_{\text {eff }}, F_{\mathrm{bol}}$, and distance

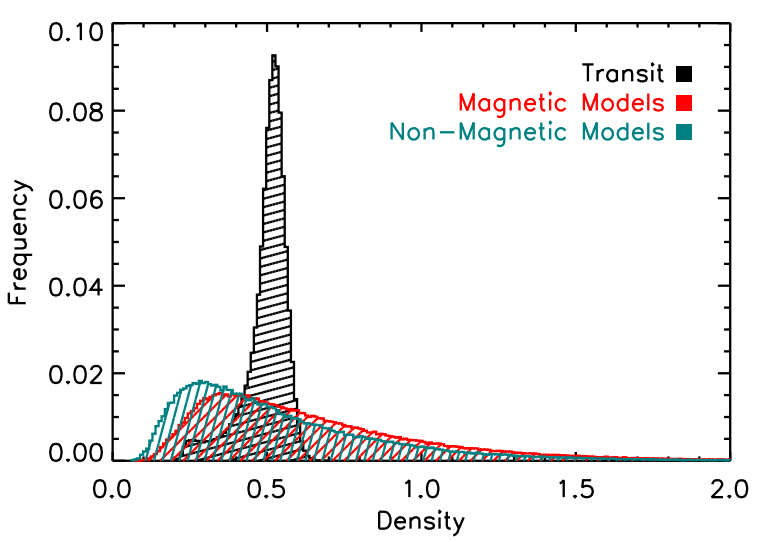

Figure 7. Stellar density $\left(\rho_{*}\right)$ from our transit fit (black, Section 3.2) assuming $e=0$ compared to that from our MCMC model comparison. Red indicates the result when using magnetic models, while teal shows the result from models without correcting for magnetic fields. Both model results shown here use uniform priors on stellar density.

uniquely determine the stellar radius, luminosity, and, when combined with the transit-fit density, the mass. Thus when the stronger distance and density priors were used the resulting radius/luminosity/mass is relatively independent of the model grid used. It is encouraging that even when using a uniform distance prior yields a distance consistent with the previously established vale for Upper Scorpius. Further, all fits to the magnetic models give an age consistent with the value estimated for high-mass members of Upper Scorpius (Pecaut et al. 2012).

Using a uniform prior on $\rho_{*}$ results in a negligible change in mass and radius, although with much larger errors. This suggests the accuracy of our stellar parameters (but not the precision) is insensitive to the assumption of zero orbital eccentricity for K2-33b. We show a comparison of the model-based and transit-fit densities in Figure 7. Because the constraints on $\rho_{*}$ are relatively weak from the model comparison alone, we cannot make definitive statements about the orbital eccentricity of the system from the data alone, and instead rely on the physical argument that a planet migrating via interaction with the disk should have $\simeq 0$ orbital eccentricity.

The non-magnetic models favor a lower mass and radius, and younger age. Magnetic models more accurately reproduce the known distance and age of Upper Scorpius. We therefore adopted parameters from the chain utilizing magnetic models with the distance prior from de Zeeuw et al. (1999) and the density prior from our transit fit, which we use for the rest of the analysis.

Rotation period: we computed the Lomb-Scargle periodogram of the K2 light curve prior to removing the stellar variability. A strong signal is apparent at 6.29 days, which we attribute to spot coverage and the rotation pe- 
Table 2. Stellar Fit Parameters

\begin{tabular}{|c|c|c|c|c|c|c|c|c|}
\hline \multirow[t]{2}{*}{ Models } & \multicolumn{2}{|c|}{ Priors } & \multicolumn{6}{|c|}{ Parameters } \\
\hline & Distance (pc) & $\rho_{*}\left(\rho_{\odot}\right)$ & $R_{*}\left(R_{\odot}\right)$ & $M_{*}\left(M_{\odot}\right)$ & $L_{*}\left(L_{\odot}\right)$ & Age (Myr) & {$[\mathrm{Fe} / \mathrm{H}]$} & Distance (pc) \\
\hline No model ${ }^{\mathrm{a}}$ & $\cdots$ & $\cdots$ & $1.02_{-0.13}^{+0.13}$ & $0.55_{-0.15}^{+0.13}$ & $0.15_{-0.03}^{+0.03}$ & $\cdots$ & $\cdots$ & $\cdots$ \\
\hline \multirow{3}{*}{ Magnetic } & $145 \pm 15$ & uniform & $1.01_{-0.17}^{+0.18}$ & $0.56_{-0.09}^{+0.09}$ & $0.14_{-0.04}^{+0.05}$ & $10.80_{-4.71}^{+8.93}$ & 0 (fixed) & $143.2_{-21.6}^{+21.0}$ \\
\hline & uniform & $0.51_{-0.07}^{+0.04 \mathrm{~b}}$ & $1.06_{-0.08}^{+0.09}$ & $0.58_{-0.10}^{+0.10}$ & $0.16_{-0.04}^{+0.05}$ & $9.31_{-1.48}^{+1.11}$ & 0 (fixed) & $152.8_{-23.5}^{+25.2}$ \\
\hline & $145 \pm 15$ & $0.51_{-0.07}^{+0.04 \mathrm{~b}}$ & $1.05_{-0.07}^{+0.07}$ & $0.56_{-0.09}^{+0.09}$ & $0.15_{-0.03}^{+0.03}$ & $9.34_{-1.26}^{+1.07}$ & 0 (fixed) & $148.0_{-15.9}^{+15.6}$ \\
\hline \multirow{3}{*}{ Non - magnetic } & $145 \pm 15$ & uniform & $0.97_{-0.16}^{+0.18}$ & $0.42_{-0.08}^{+0.10}$ & $0.14_{-0.04}^{+0.05}$ & $6.06_{-2.83}^{+6.18}$ & $0.01_{-0.14}^{+0.13}$ & $140.1_{-20.7}^{+20.1}$ \\
\hline & uniform & $0.51_{-0.07}^{+0.04 \mathrm{~b}}$ & $0.94_{-0.12}^{+0.08}$ & $0.42_{-0.09}^{+0.10}$ & $0.12_{-0.04}^{+0.04}$ & $6.53_{-1.00}^{+1.56}$ & $0.00_{-0.14}^{+0.13}$ & $135.7_{-24.3}^{+25.2}$ \\
\hline & $145 \pm 15$ & $0.51_{-0.07}^{+0.04 \mathrm{~b}}$ & $0.95_{-0.09}^{+0.07}$ & $0.43_{-0.07}^{+0.09}$ & $0.13_{-0.03}^{+0.03}$ & $6.57_{-0.96}^{+1.44}$ & $0.01_{-0.14}^{+0.13}$ & $\begin{array}{r}139.5_{-19.2}^{+18.0} \\
\end{array}$ \\
\hline
\end{tabular}

riod. We estimate an error of 0.17 days on the rotation period from the width of the peak in the periodogram power spectrum. The same period was found from the MEarth long-term monitoring (6.27 days).

Rotation velocity: We determined $v \sin i_{*}$ using our high-resolution IGRINS spectrum. We first determined the instrumental profile/resolution by fitting the telluric spectrum derived from the A0V standard (see Section 2) with a series of Gaussian profiles and assuming telluric lines have negligible intrinsic width compared to the instrument resolution. Orders with $<4$ strong $(>3 \%$ depth) telluric lines were ignored. We assumed the resolution varies linearly within an order and smoothly in between orders. Our derived instrumental broadening was $0.3-0.5 \AA$ (full-width half-max), consistent with the previously measured resolution of the spectrograph.

We then compared our IGRINS spectrum of K2-33 to the best-fit BT-SETTL model derived from the moderate resolution spectra above. We broadened the model first using the instrumental profile derived from our telluric fit, then as a function of $v \sin i_{*}$ using the IDL code lsf_rotate (Gray 1992; Hubeny \& Lanz 2011). We included seven nuisance parameters to handle normalization of the observed spectrum, small errors in wavelength calibration, and missing or overly deep/shallow lines in the atmospheric model. Orders with $\mathrm{S} / \mathrm{N}<20$ were removed. Each order was fit separately, each time adjusting $v \sin i_{*}$ and the nuisance parameters to minimize the difference between the model and IGRINS spectrum. We adopted the mean and standard error of the $v \sin i_{*}$ determinations across all orders as the final value and error: $8.2 \pm 1.8 \mathrm{~km} \mathrm{~s}^{-1}$.

Sky projected stellar inclination: The combination of $v \sin i_{*}$, rotation period, and stellar radius enabled a calculation of the (sky-projected) rotational inclination $\left(i_{*}\right)$ of K2-33. We first calculated the equatorial velocity
$V_{\text {eq }}=\frac{2 \pi R_{*}}{P_{\text {rot }}}$, where $P_{\text {rot }}$ is the stellar rotation period measured above, which yielded a velocity of $8.6 \pm 0.7 \mathrm{~km} \mathrm{~s}^{-1}$. We assumed effects of differential rotation are encapsulated in our $P_{\text {rot }}$ measurement error, although this depends on where on the star the spots are located. We converted $v \sin i_{*}$ and $V_{\text {eq }}$ to a posterior in $\cos \left(i_{*}\right)$, which handles regions of the posterior where $v \sin i_{*}>V_{\mathrm{eq}}$ (see Morton \& Winn 2014, for more details). The resulting posterior gives a lower limit on stellar inclination of $i_{*}>63^{\circ}$ at $68.3 \%(1 \sigma)$, suggesting the planetary orbit is not highly misaligned with the stellar rotation.

A summary of all derived stellar parameters and errors is given in Table 3.

\section{FALSE POSITIVE ANALYSIS}

\subsection{Background Eclipsing Binary}

We calculated a posterior probability that an unrelated, unresolved background source (i.e., an eclipsing binary) is responsible for the transit signal. The procedure is described in Gaidos et al. (2016a), and only summarized here. The Bayesian probability was calculated with a prior based on a model of the background stellar population drawn from TRILEGAL version 1.6 (Vanhollebeke et al. 2009). The likelihood is calculated from the observational constraints, i.e.,: (1) a background star must be bright enough to produce the transit signal given a maximum $50 \%$ eclipse depth; (2) the density of the star must be consistent with the measured transit duration; and (3) the star must not be visible in our NIRC2 AO imaging and NRM interferometry (Section 2).

Stars were selected from a TRILEGAL-generated synthetic catalog of 25,348 stars to $K_{p}=22$ in a field of $10 \mathrm{sq}$. deg at the coordinates of K2-33. All standard settings were used except the extinction at $\infty$ was set to $A_{V}=0.894$ based on the map of Schlegel et al. (1998). We randomly placed stars at locations in a cir- 
A Transiting Planet in Upper Scorpius

Table 3. Parameters of K2-33

\begin{tabular}{|c|c|c|}
\hline Parameter & Value & Source \\
\hline \multicolumn{3}{|c|}{ Identifiers } \\
\hline $\mathrm{K} 2-33$ & & K2 \\
\hline EPIC 205117205 & & EPIC \\
\hline USco J161014.7-191909 & & Preibisch et al. (2002) \\
\hline 2MASS J16101473-1919095 & & 2MASS \\
\hline \multicolumn{3}{|c|}{ Astrometry } \\
\hline$\alpha$ R.A. (hh:mm:ss J2000) & $16: 10: 14.73$ & EPIC \\
\hline$\delta$ Dec. (dd:mm:ss J2000) & $-19: 19: 09.38$ & EPIC \\
\hline$\mu_{\alpha}\left(\operatorname{mas} \mathrm{yr}^{-1}\right)$ & $-9.8 \pm 1.7$ & $\mathrm{UCAC} 4$ \\
\hline$\mu \delta\left({\left.\operatorname{mas~} \mathrm{yr}^{-1}\right)}\right.$ & $-24.2 \pm 1.8$ & $\mathrm{UCAC} 4$ \\
\hline \multicolumn{3}{|c|}{ Photometry } \\
\hline$B$ (mag) & $17.353 \pm 0.138$ & APASS \\
\hline$g^{\prime}(\mathrm{mag})$ & $16.386 \pm 0.076$ & APASS \\
\hline$r^{\prime}(\mathrm{mag})$ & $14.860 \pm 0.072$ & APASS \\
\hline$i^{\prime}(\mathrm{mag})$ & $13.360 \pm 0.125$ & APASS \\
\hline$z^{\prime}(\mathrm{mag})$ & $12.613 \pm 0.004$ & SDSS \\
\hline$J$ (mag) & $11.095 \pm 0.023$ & 2MASS \\
\hline$H(\mathrm{mag})$ & $10.332 \pm 0.021$ & 2MASS \\
\hline$K_{s}(\mathrm{mag})$ & $10.026 \pm 0.019$ & 2MASS \\
\hline$W 1$ (mag) & $9.890 \pm 0.023$ & ALLWISE \\
\hline$W 2(\mathrm{mag})$ & $9.762 \pm 0.021$ & ALLWISE \\
\hline$W 3$ (mag) & $9.610 \pm 0.049$ & ALLWISE \\
\hline \multicolumn{3}{|c|}{ Kinematics and Distance } \\
\hline Barycentric RV $\left(\mathrm{km} \mathrm{s}^{-1}\right)$ & $-6.70 \pm 0.15$ & This paper \\
\hline$U\left(\mathrm{~km} \mathrm{~s}^{-1}\right)$ & $-5.4 \pm 0.5$ & This paper \\
\hline$V\left(\mathrm{~km} \mathrm{~s}^{-1}\right)$ & $-15.8 \pm 2.2$ & This paper \\
\hline$W\left(\mathrm{~km} \mathrm{~s}^{-1}\right)$ & $-8.2 \pm 1.2$ & This paper \\
\hline Distance (pc) & $145 \pm 15$ & de Zeeuw et al. (1999) \\
\hline \multicolumn{3}{|c|}{ Physical Properties } \\
\hline$A_{V}$ & $0.75_{-0.18}^{+0.21}$ & This paper \\
\hline Spectral Type & $\mathrm{M} 3.3 \pm 0.5$ & This paper \\
\hline Rotation Period (days) & $6.29 \pm 0.17$ & This paper \\
\hline$T_{\text {eff }}(\mathrm{K})$ & $3540 \pm 70$ & This paper \\
\hline$F_{\mathrm{bol}}\left(10^{-10} \mathrm{erg} \mathrm{cm}^{-2} \mathrm{~s}^{-1}\right)$ & $2.25 \pm 0.26$ & This paper \\
\hline$M_{*}\left(M_{\odot}\right)$ & $0.56_{-0.09}^{+0.09}$ & This paper ${ }^{\mathrm{a}}$ \\
\hline$R_{*}\left(R_{\odot}\right)$ & $1.05_{-0.07}^{+0.07}$ & This paper ${ }^{\mathrm{a}}$ \\
\hline$L_{*}\left(L_{\odot}\right)$ & $0.15_{-0.03}^{+0.03}$ & This paper ${ }^{\mathrm{a}}$ \\
\hline Age (Myr) & $9.3_{-1.3}^{+1.1}$ & This paper ${ }^{a}$ \\
\hline$v \sin i_{*}\left(\mathrm{~km} \mathrm{~s}^{-1}\right)$ & $8.2 \pm 1.8$ & This paper \\
\hline$i_{*}$ (degrees) & $>63$ & This paper \\
\hline
\end{tabular}

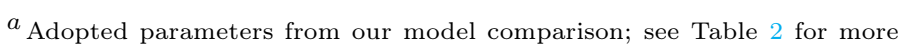
information.

cular field within 4 Kepler pixels $\left(16^{\prime \prime}\right)$ around K2-33. Stars were discarded if their position and magnitude are ruled out by the detection limits from our imaging/NRM, or they are too faint to produce the observed signal. We weighted each remaining star by the probability that an eclipsing binary with a period of 5.425 days would yield a duration consistent with our transit fit $(\simeq 4.1$ hours $)$.

The final false-positive probability is sensitive to the assumed binary eccentricity distribution. It is $<3 \times 10^{-7}$ for all reasonable choices, but is essentially zero if the orbits are near-circular, as is expected for short-period binaries; the long transit duration could only be produced by a giant star, but these are bright and ruled out by 
the lack of detections in our AO data. Background stars fainter than K2-33 will be denser dwarf stars.

\subsection{Companion Eclipsing Binary}

We next considered the possibility that the transit signal is due a physically associated companion system, i.e., a very-low mass eclipsing binary (EB). Since the maximum transit depth of an EB is $50 \%$, the contrast ratio of such a companion must be $\Delta K_{p}>5.7$, and thus absolute $M_{K_{p}}>14.0$. Such a system cannot be excluded if it is within $0.2^{\prime \prime}$ of the primary, or a projected separation of $<28 \mathrm{AU}$. According to an 11-Myr isochrone generated by the Dartmouth Stellar Evolution program, such a system would have to be substellar, i.e., a pair of eclipsing brown dwarfs or self-luminous young giant planets. Moreover splitting the light curve into even and odd transits shows that the "primary" and "secondary" transits have equal depths $(0.27 \pm 0.05$ and $0.25 \pm 0.04 \%)$, so such a system must consist of equal-mass components. This is inconsistent with the "flat-bottomed" shape of the transit light curve (Figure 3).

\subsection{Eclipsing Binary}

To confirm that the transiting body is non-stellar, we use the RVs from our IGRINS spectra to put an upper limit on the mass of K2-33b. We fit the RVs assuming a circular orbit and locking the period and argument of periapsis from the transit fit (Section 3.2) and assuming the mass derived for the host star from our model interpolation (Section 4). The RVs rule out companion masses above 3.7 Jupiter masses $\left(M_{J}\right)$ at $3 \sigma$ (Figure 8). The constraints are tighter $\left(<2.8 M_{J}\right)$ if we remove the IGRINS epoch with high telluric contamination (see Section 2). If we loosen our assumptions about the orbital eccentricity then the maximum mass increases to $5.4 M_{J}$. In all cases the RVs exclude any brown dwarf or stellar companion $\left(>13 M_{J}\right)$ with an orbital period matching the transit signal.

\subsection{Stellar Variability}

Spots and plages on the photosphere combined with stellar rotation create $1-3 \%$ variations in the light curve of K2-33. The amplitude of this variation is roughly an order of magnitude larger than the transit depth $(\simeq 0.26 \%$, Figure 3$)$. Fortunately, spots create a characteristic shape in the light curve curve (smoothly varying) and duration ( $\simeq$ half the rotation period) that differs from a transit (trapezoidal shape and a duration of hours). This makes them easy to differentiate in most stars. However, improper removal of the more complicated spot patterns on young stars can sometimes generate transit-like signals over short (days or weeks) timescales. Our BLS search identified many such systems; one of which we show in Figure 9

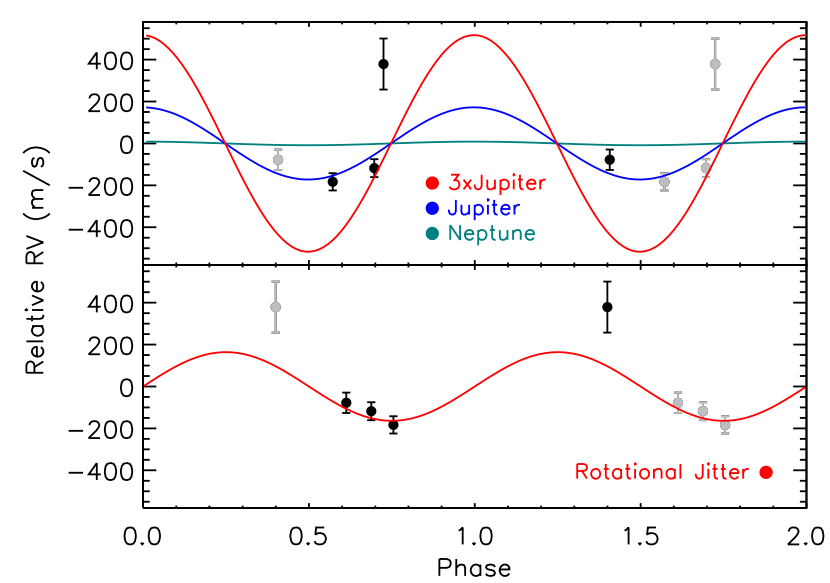

Figure 8. Radial velocities derived from our IGRINS spectra, phased to the transit-based orbital period (5.42 days, top) and stellar rotation period (6.29 days, bottom). Duplicate measurements are shown in grey. The expected RV amplitudes (assuming circular orbits) for Neptune-mass, Jupiter-mass, and $3 M_{J}$-mass planets at this orbital period are shown as teal, blue, and red lines on the top panel. An estimate of the spot-induced RV jitter, derived from the $v \sin i_{*}$ and variability in $K 2$ data, is shown in the bottom panel in red.

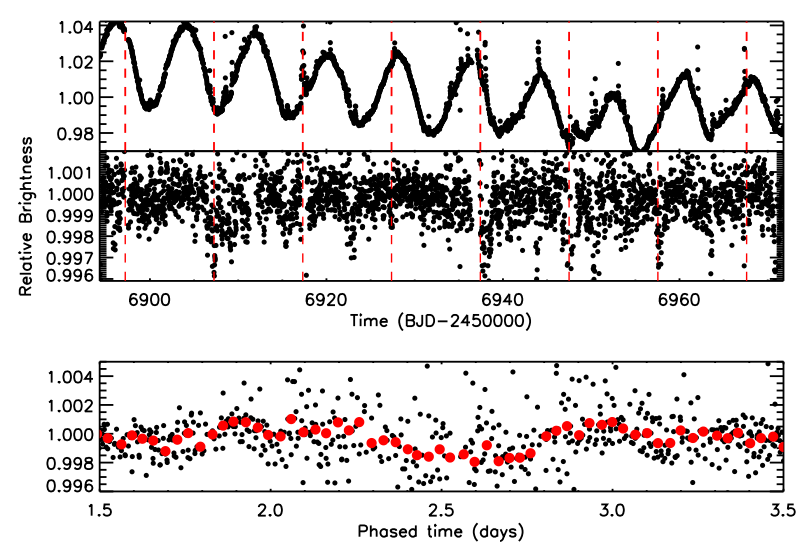

Figure 9. Light curve of a star in Upper Scorpius taken by the K2 mission, with the top two panels following the layout of Figure 1. The bottom panel shows the light curve folded to the highest power period identified from the BLS, with data binned every $20 \mathrm{~min}$ in red. Poor removal of the flares, stellar variability, and K2 drift creates systematic noise in the flattened light curve. When folded this can look like a weak transit. However, the individual transits have inconsistent depths and durations and the out-of-transit light curve contains numerous residual variations. This was identified as a candidate by our BLS search but subsequently identified as a false positive.

The combination of our MEarth and K2 light curves demonstrate that the transit signal cannot be caused by stellar variability. While spot patterns can be stable on multi-year baseline in old M dwarfs (Newton et al. 2016), spot evolution can been seen even over the 80 day observing window for Upper Scorpius members (e.g., Figure 1, 
9). If the transit signal was an artifact of stellar activity signals the transit depth and shape would change or disappear over the K2 observing window and between the K2 and MEarth observations. To test this we fit each transit individually as in Section 3.2, but locking the period to the previously derived value. We found that all transits yield consistent depths and transit durations, including MEarth data taken 1.5 years after the K2 observations. Further, stellar signals are generally wavelength dependent, and the separate fits to the $K_{2}$ $\left(\lambda_{\text {mean }} \simeq 6400 \AA\right)$ and MEarth $\left(\lambda_{\text {mean }} \simeq 8250 \AA\right)$ light curve give consistent parameters.

\subsection{Debris Disk?}

Many stars in Upper Scorpius exhibit excess emission at infrared and millimeter wavelengths indicative of cooler, circumstellar material, i.e., dusty primordial or debris disks in different stages of their evolution (Luhman \& Mamajek 2012). K2-33, may have excess emission at $24 \mu \mathrm{m}$ (measured by the MIPS instrument on Spitzer), but shows no significant excess in any of the WISE bands or the Spitzer 8 and $16 \mu \mathrm{m}$ bands (Carpenter et al. 2009; Luhman \& Mamajek 2012). We confirmed this by comparing the Spitzer measurements from Luhman \& Mamajek (2012) to our estimate of the photospheric flux (see Section 4), extrapolated to $30 \mu \mathrm{m}$ using a PHOENIX BT-SETTL model (Figure 10). This excess is consistent with the WISE upper limit at $22 \mu \mathrm{m}$ (W4 channel). This excess corresponds to that of debris disks, not an evolved disk, according to the classification of Luhman \& Mamajek (2012).

The lack of a detectable excess at wavelengths < $12 \mu \mathrm{m}$ suggests that any disk, if it exists, must lack significant material warmer than $300 \mathrm{~K}$, i.e., a central hole, or a drop in the emissivity of the grains close to the star. Using the value for $L_{*}$ estimated in Section 4, we estimated that the hole extends to $>0.35 \mathrm{AU}$. If the grains are small and not blackbody emitters, the emissivity will be higher at shorter wavelengths, thus strengthening this constraint. The central hole of any debris disk is significantly larger than the Keplerian orbit corresponding to the transit signal $(0.051 \pm 0.004 \mathrm{AU})$. This suggests that the planet and disk are physically separated and unrelated phenomena.

Disks around stars can create "dips" in the light curve as vertical structures in the disks periodically occult the host star (e.g., Cody et al. 2014). This behavior has been observed in some stars in Upper Scorpius (e.g., Ansdell et al. 2016). However, such dips are usually much deeper and are quasi-periodic or periodic, with the depth changing from dip to dip. The shapes of the dips typically do not resemble a transit; they are irregular and/or have leading or lagging tails (Ansdell et al. 2016). In contrast, the signals in the lightcurve of K2-33 are strictly

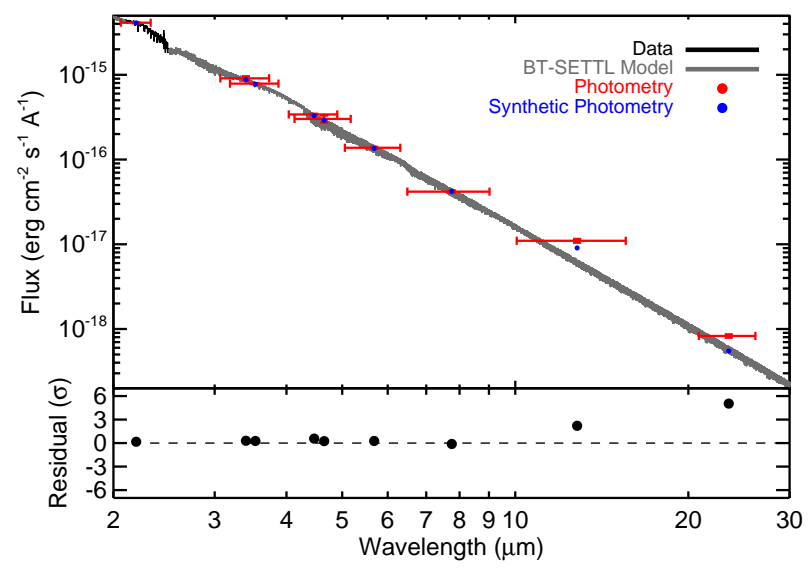

Figure 10. Spectrum of K2-33 following the format of Figure 6 , but extended into the infrared and with log scaling on both axes. Only the $24 \mu \mathrm{m}$ observation by Spitzer-MPIS shows a statistically significant excess, suggesting the presence of a cold $(<300 \mathrm{~K})$ debris disk.

periodic and transit-shaped, exhibiting no changes over the $\sim 1.5$ yr interval between the K2 and MEarth observations. Finally all such "dipper" stars exhibit significant excess emission at 12 and $24 \mu \mathrm{m}$ consistent with full or evolved disks, which is not the case for K2-33.

\section{SUMMARY \& DISCUSSION}

Young stellar associations like Upper Scorpius offer a unique view of the properties and behavior of young stars. Planets around these young stars are similarly critical probes into how planets change from formation to maturity (e.g., Aigrain et al. 2007; Quinn et al. 2012b; Bowler et al. 2015a; David et al. 2016b). In this paper we have detailed our follow-up, characterization, and confirmation of $\mathrm{K} 2-33 \mathrm{~b}$, a $5.04_{-0.37}^{+0.34} \mathrm{R}_{\oplus}$ planet orbiting at a period of 5.425 days around a star in the $\sim 11 \mathrm{Myr}$ (Pecaut et al. 2012) Upper Scorpius OB association.

In combination, K2-33's proper motions, radial velocity, and lithium and mid-IR excess in its spectrum, unambiguously indicate that $\mathrm{K} 2-33$ is a young $(<20 \mathrm{Myr})$ pre-main-sequence star associated with the Upper Scorpius association. We used moderate resolution spectra to revise the reddening, $T_{\text {eff }}$, and $F_{\text {bol }}$ determinations. We fit the K2 and MEarth transit photometry, which also yielded a precise stellar density. By interpolating these constraints onto a grid of pre-main sequence models that imitate magnetic effects on the star's internal structure, we derived a precise radius (6-7\%) and mass (16\%) for K2-33.

The data argue strongly that the transit signal is planetary in origin. Adaptive optics and radial velocities rule out a background or bound eclipsing binary as the source of the transit signal. Our MEarth transit photometry combined with the $K 2$ photometry rules out stellar variability or a disk mimicking a transit. Further, K2- 
$33 \mathrm{~b}$ exhibits none of the unusual light curve behavior of PTFO 8-8695b, the candidate whose V-shaped transit signature exhibits time-variable depth and width (van Eyken et al. 2012; Ciardi et al. 2015; Yu et al. 2015).

Few young $(<20 \mathrm{Myr})$ stars have their masses/radii determined to better than $10 \%$, with the exception of young eclipsing binaries (e.g., Kraus et al. 2015; David et al. 2016a). The precision is in part due to our careful measurement of $F_{\text {bol }}$ and $T_{\text {eff }}$, and the additional constraint from the transit lightcurve fit, which provides a stellar density accurate to $\lesssim 10 \%$. This highlights the power of transiting exoplanets to probe stellar astrophysics. While our current method relies on a stellar model, when Gaia parallaxes become available (Perryman et al. 2001; de Bruijne 2012) we can instead use transiting planets to test these models.

Our stellar density assumes that the planet has zero orbital eccentricity. This is expected for a young Neptunemass planet where recent interactions with the nascent disk have dampened eccentricity (e.g., Tanaka \& Ward 2004; Cresswell et al. 2007). However, there is a dearth of young planetary systems with known eccentricities that would be necessary to confirm this observationally. To test the sensitivity of our results to the assumption of $e=0$ we reran our stellar isochrone fits, but instead used a uniform density on $\rho_{*}$. This gave a best-fit stellar density and radius consistent with our earlier determination, but with a factor of two larger errors (see Table 2, Figure 7). Similarly, we derived a consistent stellar radius when using the Stefan-Boltzman relation, which is independent of both the transit-fit density and stellar models. We conclude that the accuracy of our stellar (and therefore planetary) radius is insensitive to this assumption.

Assuming a Neptune-like density, K2-33b will have a RV amplitude of $\sim 20 \mathrm{~ms}^{-1}$. While this is well within detection limits of current radial velocity instruments, it is smaller than the expected spot-induced RV jitter (100-200 $\left.\mathrm{m} \mathrm{s}^{-1}\right)$. Moving to the NIR can reduce this noise source, but not eliminate it (Mahmud et al. 2011; Crockett et al. 2012). Because the planet and stellar rotation periods are known from the light curve, it may be possible to fit each separately with sufficient data and baseline. K2-33 will be an excellent target for upcoming NIR radial velocity spectrographs (e.g., Quirrenbach et al. 2010; Artigau et al. 2014; Kotani et al. 2014).

As with K2-25b, K2-33b is considerably larger than close-in planets found around similar-mass stars by $\mathrm{Ke}$ pler. Most planets around M dwarfs found by Kepler are $1-2.5 R_{\oplus}$ (Morton \& Swift 2014; Mulders et al. 2015; Dressing \& Charbonneau 2015; Gaidos et al. 2016b), while $\mathrm{K} 2-33 \mathrm{~b}$ is roughly twice this size at $5.04_{-0.37}^{+0.34} \mathrm{R}_{\oplus}$. $\mathrm{K} 2-33 \mathrm{~b}$ is less of a radius outlier than K2-25b, which orbits a $0.3 M_{\odot}$ star. $\mathrm{K} 2-33$ has a mass of $0.55 M_{\odot}$ and larger planets are more common around higher mass hosts. Further, unlike with the nearby, bright, mainsequence, and photometrically well-behaved stars in the Hyades, it is not clear if our survey is sensitive to the more common $1-2 R_{\oplus}$ planets around similar-mass host stars in Upper Scorpius. However, K2-33b fits into an emerging picture that young planets are larger than their older counterparts. Mann et al. (2016) suggested these large radii could be due to the initial heat of formation as well as inflation and escape of the atmosphere under the influence of the young, active host star (e.g., Rogers et al. 2011; Ehrenreich et al. 2015).

The upper limit on K2-33b's age provided by its $\simeq 11$ Myr stellar host suggests that it either migrated inwards via disk migration or formed in-situ, as planetstar and planet-planet interactions work on much longer timescales (Fabrycky \& Tremaine 2007; Nagasawa et al. 2008), and the conditions for Kozai-Lidov evolution only begin after the disk dissipates (e.g., Martin et al. 2016). This discovery makes it unlikely that such long-term dynamical interactions are responsible for all close-in planets. However, it is difficult to draw conclusions about the dominant migration or formation mechanism for closein planets given the sample size and incomplete understanding of our transit-search pipeline's completeness.

Selection effects may be important here. It is possible that K2-33 has an atypical history of formation and migration that also made it the easiest (and hence first) planet of this age to be identified and confirmed. A full search of all young clusters and stellar associations surveyed by the $K 2$ mission, with proper treatment of detection completeness is underway. This, along with improved statistics provided by the TESS and PLATO missions, will provide an estimate of the planet occurrence rate as a function of time. Trends (or a lack of trends) in this occurrence rate could set constraints on planetary migration timescales.

During the final stages of the analysis for this paper we were informed by another team that an independent analysis of this system was about to be submitted (David et al. Nature, accepted).

We thank the anonymous referee for their thoughtful comments on the paper. The authors thank Ann Marie Cody for her help with the K2 light curves when initially identifying the transit. We also thank Charlie for his help with the data analysis and proofreading the paper.

AWM was supported through Hubble Fellowship grant 51364 awarded by the Space Telescope Science Institute, which is operated by the Association of Universities for Research in Astronomy, Inc., for NASA, under contract NAS 5-26555. This research was supported by NASA grant NNX11AC33G to EG. K.R.C. acknowledges sup- 
port provided by the NSF through grant AST-1449476. A.V. is supported by the NSF Graduate Research Fellowship, Grant No. DGE 1144152.

The MEarth Team gratefully acknowledges funding from the David and Lucille Packard Fellowship for Science and Engineering (awarded to D.C.). This material is based in part upon work supported by the National Science Foundation under grants AST-0807690, AST1109468, and AST-1004488 (Alan T. Waterman Award). This publication was made possible through the support of a grant from the John Templeton Foundation. The opinions expressed in this publication are those of the authors and do not necessarily reflect the views of the John Templeton Foundation.

The ARCoIRIS observations were conducted at Cerro Tololo Inter-American Observatory, National Optical Astronomy Observatory, which is operated by the Association of Universities for Research in Astronomy (AURA) under a cooperative agreement with the National Science Foundation. This work used the Immersion Grating Infrared Spectrograph (IGRINS) that was developed under a collaboration between the University of Texas at Austin and the Korea Astronomy and Space Science Institute (KASI) with the financial support of the US National Science Foundation under grant ASTR1229522, of the University of Texas at Austin, and of the Korean GMT Project of KASI. The IGRINS pipeline package PLP was developed by Dr. Jae-Joon Lee at Korea Astronomy and Space Science Institute and Professor Soojong Pak's team at Kyung Hee University. SNIFS on the UH $2.2-\mathrm{m}$ telescope is part of the Nearby Supernova Factory project, a scientific collab- oration among the Centre de Recherche Astronomique de Lyon, Institut de Physique Nucléaire de Lyon, Laboratoire de Physique Nucléaire et des Hautes Energies, Lawrence Berkeley National Laboratory, Yale University, University of Bonn, Max Planck Institute for Astrophysics, Tsinghua Center for Astrophysics, and the Centre de Physique des Particules de Marseille. Some of the data presented in this paper were obtained from the Mikulski Archive for Space Telescopes (MAST). STScI is operated by the Association of Universities for Research in Astronomy, Inc., under NASA contract NAS5-26555. Support for MAST for non-HST data is provided by the NASA Office of Space Science via grant NNX09AF08G and by other grants and contracts. This research was made possible through the use of the AAVSO Photometric All-Sky Survey (APASS), funded by the Robert Martin Ayers Sciences Fund.

The authors wish to recognize and acknowledge the very significant cultural role and reverence that the summit of Maunakea has always had within the indigenous Hawaiian community. We are most fortunate to have the opportunity to conduct observations from this mountain.

Facilities: Blanco (ARCoIRIS), UH:2.2m (SNIFS), Keck:II (NIRC2), Smith (IGRINS), Kepler

Software: Spextool (Cushing et al. 2004), xtellcor (Vacca et al. 2003), IGRINS pipeline (Lee 2015), ARCoIRIS Spextool, batman (Kreidberg 2015), emcee (Foreman-Mackey et al. 2013)

\section{REFERENCES}

Ahn, C. P., Alexandroff, R., Allende Prieto, C., et al. 2012, ApJS, 203, 21

Aigrain, S., Hodgkin, S., Irwin, J., et al. 2007, MNRAS, 375, 29

Aldering, G., Adam, G., Antilogus, P., et al. 2002, in Society of Photo-Optical Instrumentation Engineers (SPIE) Confer 61

Allard, F., Homeier, D., \& Freytag, B. 2011, in Astronomical Society of the Pacific Conference Series, Vol. 448, 16th Cambridge Workshop on Cool Stars, Stellar Systems, and the Sun, ed. C. Johns-Krull, M. K. Browning, \& A. A. West, 91

Allard, F., Homeier, D., Freytag, B., \& Sharp, C. M. 2012, in EAS Publications Series, Vol. 57, EAS Publications Series, 3

Ansdell, M., Gaidos, E., Rappaport, S. A., et al. 2016, ApJ, 816, 69

Artigau, É., Kouach, D., Donati, J.-F., et al. 2014, in Society of Photo-Optical Instrumentation Engineers (SPIE)

Becker, J. C., Vanderburg, A., Adams, F. C., Rappaport, S. A., \& Schwengeler, H. M. 2015, ApJL, 812, L18

Berta, Z. K., Charbonneau, D., Désert, J.-M., et al. 2012, ApJ, 747, 35

Bowler, B. P., Liu, M. C., Shkolnik, E. L., \& Tamura, M. 2015a, ApJS, 216, 7
Bowler, B. P., Shkolnik, E. L., Liu, M. C., et al. 2015b, ApJ, 806, 62

Boyajian, T. S., von Braun, K., van Belle, G., et al. 2012, ApJ, 757, 112

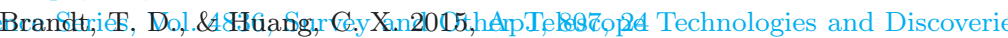

Bubar, E. J., Schaeuble, M., King, J. R., Mamajek, E. E., \& Stauffer, J. R. 2011, AJ, 142, 180

Cardelli, J. A., Clayton, G. C., \& Mathis, J. S. 1989, ApJ, 345, 245

Carpenter, J. M., Bouwman, J., Mamajek, E. E., et al. 2009, ApJS, 181, 197

Chatterjee, S., Ford, E. B., Matsumura, S., \& Rasio, F. A. 2008, ApJ, 686, 580

Chen, Y., Girardi, L., Bressan, A., et al. 2014, MNRAS, 444, 2525

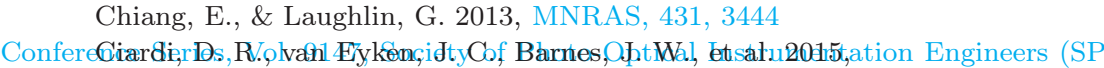
ApJ, 809, 42

Cody, A. M., Stauffer, J., Baglin, A., et al. 2014, AJ, 147, 82

Cohen, M., Wheaton, W. A., \& Megeath, S. T. 2003, AJ, 126, 1090

Cresswell, P., Dirksen, G., Kley, W., \& Nelson, R. P. 2007, A\&A, 473, 329 
Crockett, C. J., Mahmud, N. I., Prato, L., et al. 2012 ApJ, 761, 164

Cushing, M. C., Vacca, W. D., \& Rayner, J. T. 2004, PASP, 116, 362

Dahm, S. E. 2015, ApJ, 813, 108

David, T. J., Hillenbrand, L. A., Cody, A. M., Carpenter, J. M., \& Howard, A. W. 2016a, ApJ, 816, 21

David, T. J., Conroy, K. E., Hillenbrand, L. A., et al. 2016b, AJ, 151, 112

de Bruijne, J. H. J. 2012, Ap\&SS, 341, 31

de Zeeuw, P. T., Hoogerwerf, R., de Bruijne, J. H. J., Brown, A. G. A., \& Blaauw, A. 1999, AJ, 117, 354

Dittmann, J. A., Irwin, J. M., Charbonneau, D., \& Newton, E. R. 2016, ApJ, 818, 153

Dotter, A., Chaboyer, B., Jevremović, D., et al. 2008, ApJS, 178, 89

Dressing, C. D., \& Charbonneau, D. 2015, ApJ, 807, 45

Ehrenreich, D., Bourrier, V., Wheatley, P. J., et al. 2015, Nature, 522, 459

Fabrycky, D., \& Tremaine, S. 2007, ApJ, 669, 1298

Feiden, G. A. 2016, ArXiv e-prints, arXiv:1604.08036 [astro-ph.SR]

Feiden, G. A., \& Chaboyer, B. 2012, ApJ, 761, 30

—. 2013, ApJ, 779, 183

Ford, E. B., \& Rasio, F. A. 2006, ApJL, 638, L45

Foreman-Mackey, D., Hogg, D. W., Lang, D., \& Goodman, J. 2013, PASP, 125, 306

Fressin, F., Torres, G., Charbonneau, D., et al. 2013, ApJ, 766, 81

Gaidos, E., Mann, A. W., \& Ansdell, M. 2016a, ApJ, 817, 50

Gaidos, E., Mann, A. W., Kraus, A. L., \& Ireland, M. 2016b, MNRAS, 457, 2877

Gaidos, E., Mann, A. W., Lépine, S., et al. 2014, MNRAS, 443, 2561

Gray, D. F. 1992, The observation and analysis of stellar photospheres., Vol. 10 (Camb. Astrophys. Ser)

Henden, A. A., Levine, S. E., Terrell, D., Smith, T. C., \& Welch, D. 2012, Journal of the American Association of Variable Star Observers (JAAVSO), 40, 430

Herczeg, G. J., \& Hillenbrand, L. A. 2014, ApJ, 786, 97

Howard, A. W., Marcy, G. W., Johnson, J. A., et al. 2010, Science, 330, 653

Howell, S. B., Sobeck, C., Haas, M., et al. 2014, PASP, 126, 398

Hubeny, I., \& Lanz, T. 2011, Synspec: General Spectrum Synthesis Program, Astrophysics Source Code Library, ascl:1109.022

Husser, T.-O., Wende-von Berg, S., Dreizler, S., et al. 2013, A\&A, 553, A6

Ida, S., \& Lin, D. N. C. 2008, ApJ, 673, 487

Irwin, J., Irwin, M., Aigrain, S., et al. 2007, MNRAS, 375, 1449

Irwin, J. M., Berta-Thompson, Z. K., Charbonneau, D., et al. 2015, in Cambridge Workshop on Cool Stars, Stellar Systems, and the Sun, Vol. 18, 18th Cambridge Workshop on Cool Stars, Stellar Systems, and the Sun, ed. G. T. van Belle \& H. C. Harris, 767

Janes, K. 1996, J. Geophys. Res., 101, 14853

Jarrett, T. H., Cohen, M., Masci, F., et al. 2011, ApJ, 735, 112

Kenworthy, M. A., Lacour, S., Kraus, A., et al. 2015, MNRAS, 446, 411

Kenyon, S. J., Gómez, M., \& Whitney, B. A. 2008, Low Mass Star Formation in the Taurus-Auriga Clouds, ed. B. Reipurth, Vol. 1 (Astronomical Society of the Pacific), 405

Kipping, D. M. 2010, MNRAS, 408, 1758

-. 2013, MNRAS, 435, 2152

Kotani, T., Tamura, M., Suto, H., et al. 2014, in Society of Photo-Optical Instrumentation Engineers (SPIE) ConfereR 14
Kovács, G., Zucker, S., \& Mazeh, T. 2002, A\&A, 391, 369

Kraus, A. L., Cody, A. M., Covey, K. R., et al. 2015, ApJ, 807, 3

Kraus, A. L., \& Hillenbrand, L. A. 2008, ApJL, 686, L111

Kraus, A. L., Ireland, M. J., Martinache, F., \& Lloyd, J. P. 2008, ApJ, 679, 762

Kreidberg, L. 2015, PASP, 127, 1161

Lantz, B., Aldering, G., Antilogus, P., et al. 2004,

in Society of Photo-Optical Instrumentation Engineers (SPIE) Conference S 146

Le Bouquin, J.-B., \& Absil, O. 2012, A\&A, 541, A89

Lee, J.-J. 2015, plp: Version 2.0

Lubow, S. H., \& Ida, S. 2010, ArXiv e-prints, arXiv: 1004.4137 [astro-ph.EP]

Luhman, K. L., \& Mamajek, E. E. 2012, ApJ, 758, 31

Mahmud, N. I., Crockett, C. J., Johns-Krull, C. M., et al. 2011, ApJ, 736, 123

Mamajek, E. E., Pecaut, M. J., Nguyen, D. C., \& Bubar, E. J. 2013, in Protostars and Planets VI Posters

Mamajek, E. E., Quillen, A. C., Pecaut, M. J., et al. 2012, AJ, 143, 72

Mandel, K., \& Agol, E. 2002, ApJL, 580, L171

Mann, A. W., Feiden, G. A., Gaidos, E., Boyajian, T., \& von Braun, K. 2015, ApJ, 804, 64

Mann, A. W., Gaidos, E., \& Ansdell, M. 2013, ApJ, 779, 188

Mann, A. W., \& von Braun, K. 2015, PASP, 127, 102

Mann, A. W., Gaidos, E., Mace, G. N., et al. 2016, ApJ, 818, 46

Markwardt, C. B. 2009, in Astronomical Society of the Pacific Conference Series, Vol. 411, Astronomical Data Analysis Software and Systems XVIII, ed. D. A. Bohlender, D. Durand, \& P. Dowler, 251

Martin, R. G., Lubow, S. H., Nixon, C., \& Armitage, P. J. 2016, MNRAS, arXiv:1603.03135 [astro-ph.EP]

Morton, T. D., \& Swift, J. 2014, ApJ, 791, 10

Morton, T. D., \& Winn, J. N. 2014, ApJ, 796, 47

Mulders, G. D., Pascucci, I., \& Apai, D. 2015, ApJ, 798, 112

Nagasawa, M., Ida, S., \& Bessho, T. 2008, ApJ, 678, 498

Newton, E. R., Irwin, J., Charbonneau, D., et al. 2016, ApJ, 821, 93

Nutzman, P., \& Charbonneau, D. 2008, PASP, 120, 317

Ogihara, M., Morbidelli, A., \& Guillot, T. 2015, A\&A, 578, A36

Park, C., Jaffe, D. T., Yuk, I.-S., et al. 2014, in Society of Photo-Optical Instrumentation Engineers (SPIE) Conference S 1

Parviainen, H., \& Aigrain, S. 2015, MNRAS, 453, 3821

Paulson, D. B., Cochran, W. D., \& Hatzes, A. P. 2004, AJ, 127, 3579

Pecaut, M. J., Mamajek, E. E., \& Bubar, E. J. 2012, ApJ, 746, 154

Perryman, M. A. C., de Boer, K. S., Gilmore, G., et al. 2001, A\&A, 369, 339

Preibisch, T., Brown, A. G. A., Bridges, T., Guenther, E., \& Zinnecker, H. 2002, AJ, 124, 404

Preibisch, T., Guenther, E., \& Zinnecker, H. 2001, AJ, 121, 1040

Quinn, S. N., Bakos, G. Á., Hartman, J., et al. 2012a, ApJ, 745, 80

Quinn, S. N., White, R. J., Latham, D. W., et al. 2012b, ApJL, 756, L33

-. 2014, ApJ, 787, 27

Quirrenbach, A., Amado, P. J., Mandel, H., et al. 2010, in Society of Photo-Optical Instrumentation Engineers (SPIE) Conference S

Raymond, S. N., \& Cossou, C. 2014, MNRAS, 440, L11

Rizzuto, A. C., Ireland, M. J., Dupuy, T. J., \& Kraus, A. L. 2016, ApJ, 817, 164

Rizzuto, A. C., Ireland, M. J., \& Kraus, A. L. 2015, MNRAS, 448, 2737

RizztitoieA. VC.,. Irelland,oMety., o\& Robertsphi,cal. Gist201hentation Engineers (SP MNRAS, 416, 3108 
Rogers, L. A., Bodenheimer, P., Lissauer, J. J., \& Seager, S. 2011, ApJ, 738, 59

Schlaufman, K. C., Lin, D. N. C., \& Ida, S. 2009, ApJ, 691, 1322

Schlawin, E., Herter, T. L., Henderson, C., et al. 2014, in Proc. SPIE, Vol. 9147, Ground-based and Airborne Instrumentatio $91472 \mathrm{H}$

Schlegel, D. J., Finkbeiner, D. P., \& Davis, M. 1998, ApJ, 500, 525

Skrutskie, M. F., Cutri, R. M., Stiening, R., et al. 2006, AJ, 131, 1163

Slesnick, C. L., Carpenter, J. M., \& Hillenbrand, L. A. 2006, AJ, 131, 3016

Tanaka, H., \& Ward, W. R. 2004, ApJ, 602, 388

Vacca, W. D., Cushing, M. C., \& Rayner, J. T. 2003, PASP, 115, 389

-. 2004, PASP, 116, 352 van Eyken, J. C., Ciardi, D. R., von Braun, K., et al. 2012, ApJ, 755, 42

Vanderburg, A., \& Johnson, J. A. 2014, PASP, 126, 948

Vanderburg, A., Latham, D. W., Buchhave, L. A., et al. 2016, Ap.JS, 222, 14

Vanhollebeke, £., Groenewegen, M. A. T., \& Girardi, L. 2009, A\&A, 498, 95

Ward, W. R. 1997, ApJL, 482, L211

Wizinowich, P., Acton, D. S., Shelton, C., et al. 2000, PASP, 112, 315

Wright, E. L., Eisenhardt, P. R. M., Mainzer, A. K., et al. 2010, AJ, 140, 1868

Wu, Y., \& Murray, N. 2003, ApJ, 589, 605

Yelda, S., Lu, J. R., Ghez, A. M., et al. 2010, ApJ, 725, 331

Yu, L., Winn, J. N., Gillon, M., et al. 2015, ApJ, 812, 48

Zacharias, N., Finch, C. T., Girard, T. M., et al. 2012, VizieR Online Data Catalog, 1322, 0 